\title{
Storytelling in Unternehmenskontexten
}

\author{
Karin Thier
}

\section{Geschichten und Erzäblungen in Organisationen}

In allen Epochen und Kulturen waren und sind es Geschichten, die der Welt Bedeutung gaben, Bindung zwischen Menschen erzeugten und Ordnung in Gesellschaften brachten: „Stories are tribal codes for establishing order in all societies. They are rules for family relationships, as found in Greek mythology, as well as in IBM's unwritten codes for supervisor and employee interaction" (Hansen/ Kahnweiler 1993: 1391). Und wie in allen Bereichen des Lebens so sind auch Menschen in Unternehmen, laut dem Organisationsforscher Boje, „natural born storytellers" (1994: 434). Sie erzählen sich z. B. Geschichten über erfolgreiche Projekte, über dramatische Fehlschläge oder über die unrealisierbaren Ideen des neuen Chefs.

Wann aber sprechen wir eigentlich von „organisationalen Geschichten“ bzw. Geschichten in Unternehmen? Im Grunde spricht man immer dann von einer (organisationalen) Geschichte, wenn folgende drei Merkmale gegeben sind:

1. eine Ausgangslage

2. ein Ereignis

3. eine Konsequenz

Ein Ereignis alleine macht also noch keine Geschichte aus. Erst durch eine logische Handlungsfolge (Ausgangslage $\rightarrow$ Konsequenz) wird aus einem Ereignis ein bedeutungsvolles Ganzes und man spricht von einer Geschichte. Wichtig ist eine chronologisch sinnvolle Reihenfolge von Ereignissen (Czariniawska 1998). Damit sind die Fragen nach dem ,Wie ${ }^{6}$ und dem ,Was ${ }^{6}$ in einer Geschichte beantwortet.

Nicht vollständig sind Geschichten jedoch ohne Charaktere und Handlungen, die diese ausführen. Die Charaktere geben uns Auskunft über das, Wer ${ }^{6}$ und die Handlung gibt Aufschluss über das, Warum‘ (Mitroff 1983).

Im Alltag von Unternehmen werden aber nur selten vollständige Geschichten erzählt, sondern oftmals lediglich kleine, für den eingeweihten, informierten Zuhörer aber noch verständliche und nachvollziehbare Teile einer ganzen Geschichte. Oft genügt ein Hinweis, wie: „Erinnerst du dich, was unser Boss dazu gesagt hat?“", um die dahinterliegende Geschichte beim Zuhörer zu aktivieren (vgl. Boje 1991).

Unterschieden werden muss auch zwischen Geschichten, die zur Unterhaltung bzw. die, ohne einen bestimmten Zweck zu verfolgen, in Unternehmen erzählt werden (z. B. bei Betriebsfeiern und in Kaffeeküchen), und Geschichten, die strategisch vom Management erzählt und eingesetzt werden, um z. B. von einer Idee $\mathrm{zu}$ überzeugen oder Veränderungsprozesse zu unterstützen. Schank unterscheidet 
zwischen drei Gründen, warum Menschen überhaupt Geschichten erzählen (vgl. Schank 1990):

1. „Me-Goals“ - mit der erzählten Geschichte soll ein persönliches Ziel erreicht werden (z. B. Aufmerksamkeit erregen, Bestätigung bekommen).

2. „Your-Goals“ - mit der erzählten Geschichte soll ein bestimmter Effekt beim Zuhörer erzielt werden (z. B. Informationen übertragen, jemand in eine andere Richtung lenken).

3. „Conversational Goals“ - mit der erzählten Geschichte soll die Konversation beeinflusst werden. Hier geht es darum, mit Hilfe der Geschichte eine Konversation hinsichtlich eines bestimmten Themas zu eröffnen, in Gang zu halten oder das Thema mittels einer neuen Geschichte zu ändern.

Als gemeinsames Merkmal aller Geschichten in Organisationen sehen Reinmann-Rothmeier/Vohle (2002) die implizite oder explizite Absicht an, konkrete Vorstellungen zu provozieren, neue Denkmöglichkeiten aufzuzeigen und zur Veränderung des Handelns anzuregen. Zudem sehen sie eine Reihe von Merkmalen, die Geschichten von anderen Formen der Darstellung abgrenzen (348):

- Sequentielle Struktur: Geschichten beinhalten ein dramaturgisches Muster mit einer bestimmten Handlungsabfolge, das den Zuhörer durch die Inhalte führt.

- Kontext- und Personenbezug: Geschichten basieren auf konkreten Situationen und Charakteren und setzen auf situationalen und personalen Detailreichtum.

- Botschaft: Geschichten wollen belehren und bewerten.

- Interpretationsoffenheit: Geschichten lassen mehr als eine Interpretation zu und sind mehrdeutig.

- Literarische Elemente: Geschichten sind voll von Metaphern, Analogien und assoziationsreichen Begriffen.

- Identifikationsmuster: Geschichten vermitteln das Gefühl der Zugehörigkeit und stiften Gemeinschaft.

\section{Welche Geschichtstypen und -formen lassen sich in Unternehmen finden?}

Laut dem Organisationswissenschaftler Nymark (2000) lassen sich in Organisationen generell zwei unterschiedliche Formen von Geschichten finden: formale und informelle Geschichten. Nymark macht die Unterscheidung an der Aufgabe fest, die eine Geschichte im Unternehmen erfüllen soll. Während informelle Geschichten die Aufgabe haben, unter Mitarbeitern einen Sinn und eine Bedeutung für bestimmte Ereignisse zu stiften, werden formale Geschichten hingegen vom Management gezielt eingesetzt, um Werte und Visionen zu vermitteln.

Als wichtigste Unterscheidungsform von Geschichten in Organisationen sehen Reimann-Rothmeier/Vohle (2001) die Tatsache, ob es sich um eine authentische Geschichte handelt, die reale Geschehnisse wiedergibt, wie dies Fallgeschichten 
und Anekdoten tun, oder um eine analoge Geschichte, die einen gedanklichen Sprung von der Geschichte zum eigentlichen Kontext macht. Hierunter fallen Märchen und Gleichnisse. Sie betonen aber, dass es in Organisationen darüber hinaus ganz unterschiedliche Formen von Geschichten gebe und man immer genau nachfragen müsse, um was für eine Geschichte es sich handle.

Martin et al. (1983), die im Rahmen ihrer Forschungsarbeiten zahlreiche Geschichten in und über Organisationen sammelten und kategorisierten, unterteilen die von ihnen entdeckten Geschichten hingegen nach verschiedenen, sich häufig wiederholenden Inhaltsmustern. Insgesamt entdeckten die Autoren sieben sich immer wieder in verschiedenen Formen wiederholende Geschichtstypen in Organisationen, die sie folgendermaßen beschreiben (440 ff.):

1. „Was ist zu tun, wenn eine Person der oberen Ebene einen Regelverstoß begeht?" - Bei diesen Geschichten spielen immer ein Mitarbeiter der obersten Ebene und ein Mitarbeiter einer unteren Ebene mit, wobei der Mitarbeiter der obersten Ebene einen Regelverstoß begeht, der von dem Mitarbeiter der unteren Ebene entdeckt und geahndet wird. Von großer Bedeutung sind hier der Statusunterschied und die Reaktion des Statushöheren.

2. „Ist unser Boss auch menschlich?“ - Die zentrale Rolle bei diesem Geschichtstyp spielt eine hochrangige Persönlichkeit einer Organisation (z. B. Gründer oder Geschäftsführer). Anhand eines Ereignisses oder einer Verhaltensweise wird aufgezeigt, ob sich die Person in bestimmten Situationen human verhält oder nicht, z. B. wenn der Geschäftsführer eines Elektrobetriebes in Engpässen selbst Reparaturen durchführt.

3. „Kann ein einfacher Mitarbeiter eine Führungsposition erhalten?“ - Hier geht es um die Möglichkeiten und Aufstiegschancen, die Mitarbeiter in einer Organisation auch bei eher schlechten Ausgangspositionen haben. Im Mittelpunkt steht dabei ein Mitarbeiter mit niedrigem Status, der es schafft (oder auch nicht), eine hohe Position zu erlangen.

Diese ersten drei Geschichtstypen handeln laut Martin et al. (1983) von Statusunterschieden und wie damit in Organisationen umgegangen wird (448).

4. „Werde ich schnell gefeuert?" - Hierbei geht es darum, wie in Organisationen mit Mitarbeiterentlassungen umgegangen wird und wie sehr sich das Unternehmen bemüht, dem entgegenzuwirken. Im Zentrum stehen hier meist Personengruppen, die von Entlassung betroffen sind, und andererseits Entscheider im Unternehmen.

5. „Hilft die Organisation bei Umzügen und Arbeitsplatzwechseln?“ - Im Mittelpunkt stehen hier die persönlichen Schwierigkeiten von Mitarbeitern, die von der Organisation dazu aufgefordert werden, in eine neue Stadt oder ein neues Land zu ziehen und das Entgegenkommen der Organisation, es dem Mitarbeiter so einfach wie möglich zu machen.

6. „Wie reagiert der Boss auf Fehler?“ - Zwei Charaktere sind hier zentral: ein Mitarbeiter, der einen schwerwiegenden Fehler begeht und ein Vorgesetzter. 
An der Reaktion des Vorgesetzten kann der Zuhörer ablesen, wie in der Organisation Fehler geahndet werden, z. B. ob sich der Vorgesetzte vor einem Kunden hinter seinen Mitarbeiter stellen wird.

In den drei letzten Geschichtstypen geht es um Unsicherheiten bzw. Sicherheit, denen ein Mitarbeiter in der Organisation begegnen kann und wie die Organisation den Mitarbeiter in dieser Situation schützt bzw. ihn ihr ausliefert.

7. „Wie wird in der Organisation mit Problemen und Hindernissen umgegangen?" - Dieser Geschichtstyp ist laut Martin et al. (1983) am häufigsten verbreitet und es gibt viele Variationen davon (444). Es kann sich hierbei um den Umgang der Organisation mit externen Problemen (z. B. ein Feuer in der Werkshalle), mit technischen Problemen (z. B. Maschinenausfall) oder mit durch Mitarbeiter verursachte Probleme handeln. Die Hauptcharaktere in diesen Geschichten sind aber immer die Mitarbeiter. Bei diesem Geschichtstyp geht es um den Grad der vorhandenen Kontrolle in einer Organisation und wie mit unvorhergesehenen Hindernissen umgegangen wird.

Im Gegensatz zu den Forschungsarbeiten von Martin et al. (1983), die versuchten, alle in Unternehmen auffindbaren Geschichten bei ihrer Aufzählung zu berücksichtigen, konzentriert sich der Unternehmensberater Owen (1987) bei seiner eher auf Erfahrungen aus der Praxis beruhenden Einteilung von Geschichten nur auf ,zentrale Geschichten' in Organisationen. Er stellt die These auf, dass es in jeder Organisation, egal wie klein oder groß diese sei, immer ein paar zentrale Geschichten gäbe, welche die Realitätswahrnehmung maßgeblich determinierten. Diese zentralen Geschichten seien es, durch die die Vergangenheit, die Gegenwart und die Zukunft einer Organisation erfahren werden könne. Als zentrale Geschichten können Geschichten aber nur dann gelten, wenn sie von der Mehrheit der Mitglieder gekannt und erzählt würden.

Die von Owen beschriebenen zentralen Geschichten lassen sich ebenfalls in verschiedene Arten unterteilen (vgl. Bonsen 2000: 87 ff.):

- ,Schöpfungsgeschichten': Dies sind Geschichten, die sich um die Gründung bzw. um Gründungsväter und -mütter von Organisationen ranken und viel über die Richtung, die Werte und Glaubenssätze von Organisationen aussagen.

- ,Saure' Geschichten: Darunter versteht man Geschichten über Misserfolge und Niederlagen in Organisationen. Solche Geschichten beinhalten zwar fast immer eine Moral und können dazu anspornen, aus den gemachten Fehlern zu lernen, Bonsen sieht in ihnen jedoch auch die Gefahr der Demoralisierung und Vergiftung der Atmosphäre.

- ,Wiederauferstehungsgeschichten': Gemeint sind Geschichten einer überwundenen Krise, wie z. B. ein Beinahe-Bankrott.

- ,Transformationsgeschichten': Das sind Geschichten, z. B. über Fusionen oder Änderungen der Organisationsform. 
- ,Inspirierende‘ Geschichten: Hierunter sind Geschichten über positive Ereignisse oder Verhaltensweisen gemeint, d. h. Geschichten mit gutem Ausgang, die dazu anregen, das Erzählte zu übernehmen.

Für den Kommunikationsforscher Browning (1991) steht bei der Einteilung von Geschichten im Mittelpunkt, dass diese die Organisationen dabei unterstützen, Strukturen zu kreieren. Browning beschreibt drei Geschichtstypen, die dies tun (60 ff.):

1. ,Geschichten über den Aufstieg': Hierunter fallen oft Gründungsgeschichten. Im Mittelpunkt steht eine zentrale Person: der Star.

2. ,Geschichten über Rück- und Niederschläge‘: Diese Geschichten erzählen dramatische Niederlagen, wie z. B. Entlassungen. Diese sind wichtig, weil Rückund Niederschläge oft zu Veränderungen in Organisationen führen können bzw. diese erklären. Die ersten beiden Geschichtstypen sind laut Browning in eher hierarchischen Organisationen zu finden.

3. ,Geschichten über gute Zeiten': Diese Geschichten sind die schwierigsten, da ihnen oft nichts Dramatisches anhaftet. Sie sind aber wichtig, da sie die Kultur von Organisationen vermitteln. Der dritte Geschichtstyp findet sich verstärkt bei Organisationen mit eher flacheren Hierarchien und Strukturen.

Auch der Organisationstheoretiker Gabriel (2000) fand verschiedene Geschichtsarten in Organisationen, die er nach ihrer dichterischen Form einteilte („poetic mo-

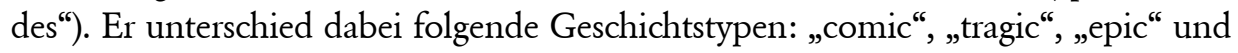
„romantic“ (61 ff.). Im Rahmen seiner Untersuchungen stellte Gabriel außerdem fest, dass deutlich mehr Geschichten in Organisationen das Negative akzentuierten. Vor allem Geschichten, die die Gegenwart repräsentierten, seien eher negativ. Vergangenes hingegen werde tendenziell eher positiv dargestellt. Generell lassen sich in jeder Organisation ganz unterschiedliche Geschichtsarten und -typen finden.

Aber wann bzw. unter welchen Umständen entstehen und verändern sich überhaupt Geschichten in Unternehmen? Der Berater Bonsen vermutet, dass die meisten Geschichten in Organisationen aus einschneidenden Veränderungsvorgängen oder großen Erfolgen bzw. Misserfolgen hervorgingen. Denn dies sei der „Stoff“, aus dem Geschichten gewoben würden (2000: 89).

Der Organisationstheoretiker Wilkins (1983) fand heraus, dass sich Geschichten in Unternehmen am besten unter zwei Voraussetzungen entwickeln: Zum einen, wenn Entscheider immer wieder bestimmte Ideen, Visionen oder Systeme betonten und zum anderen, wenn die Zugehörigkeitsdauer von Mitgliedern der Organisation relativ hoch sei (86). Unter diesen Bedingungen hätten die Mitglieder genug Zeit und Material, um Geschichten zu entwickeln und weiterzugeben. Wilkins fand daneben in Organisationen mit einer langen Mitarbeiterzugehörigkeit deutlich mehr Geschichten als in Organisationen mit häufig wechselndem Mitarbeiterstab.

Darüber hinaus stellte Nymark (2000) fest, dass sich Geschichten in Unternehmen über die Zeit weiterentwickeln und verändern können und zwar immer 
dann, wenn neue Aspekte auftauchten oder neue Inputs von Personen hinzugefügt würden. Eine Geschichte im Unternehmen impliziere eher etwas Statisches, während das Erzählen von Geschichten bewusst als Prozess zu verstehen sei. So kann sich eine Geschichte in einer Organisation über die Zeit durch den Input verschiedener Mitglieder immer weiterentwickeln.

Die Entwicklung von Geschichten in Unternehmen ist aber ein Prozess, der unberensst durchlaufen wird. Nymark behauptet, dass die einzigen bewusst entwickelten Geschichten in Unternehmen Geschichten seien, die vom Management initiiert würden, um ein bestimmtes Image der Organisation zu kreieren (48).

$\mathrm{Ob}$ man nun nach wiederkehrenden Inhaltsmustern, nach für das Unternehmen ,zentralen' Geschichten oder verschiedenen dichterischen Formen sucht, wenn man bei Geschichten in Organisationen betrachtet bzw. untersucht, ob sie sich aufgrund markanter Veränderungsprozesse oder der langen Zugehörigkeitsdauer der Mitarbeiter entwickelt haben, spielt letztlich unter Gesichtspunkten der Nützlichkeit von Geschichten für Organisationen eine eher zweitrangige Rolle. Es zeigt die unterschiedlichen Ausgangspunkte und Herangehensweisen auf, unter denen man organisationale Geschichten betrachten kann.

Bevor die verschiedenen Rollen und Funktionen von organisationalen Geschichten aufgezählt werden, soll angemerkt werden, dass es zwar schon einige wissenschaftliche Auseinandersetzungen mit diesem Thema gibt, aber dass die folgenden Aussagen nicht immer aufgrund fundierter Forschungsarbeiten getroffen werden konnten. Teilweise berufen sich die hier genannten Autoren eher auf Beobachtungen bei der praktischen Arbeit mit Geschichten in Organisationen. (vgl. auch Thier 2003).

Schon Anfang der 80er Jahre betonte der Organisationsforscher Wilkins (1983) die Bedeutung von Geschichten für den sozialen Zusammenhalt in Organisationen. Bei Untersuchungen in zwei verschiedenen Organisationen fand er heraus, dass in der Organisation, in der eine größere Bindung der Mitarbeiter an ihr Unternehmen vorhanden war, deutlich mehr Geschichten unter den Mitarbeitern erzählt wurden. Wilkins schloss daraus, dass eine Beziehung zwischen der Bindung zu einem Unternehmen und den dort erzählten Geschichten bestehen muss. Auch die Organisationspsychologin Martin (1992) hat in ihren Studien immer wieder darauf hingewiesen, dass über Geschichten mehr Bindung zu einer Organisation erzeugt werden kann als mit irgendeiner anderen Form, wie z. B. Statistiken oder Aussagen über Werte in Unternehmen. Laut Peters/Waterman (1982) wird mittels erzählter Geschichten sogar eine Art gemeinsame Fähigkeit (corporate excellence) und gemeinsame Kultur (corporate culture) erzeugt.

Boje nimmt an, dass Geschichten über bestimmte Ereignisse in Unternehmen dazu genutzt werden, das soziale Gedächtnis am Leben zu erhalten und Unternehmen davor zu bewahren, historische Fehler zu wiederholen (1991, 1994: 35). 
Allerdings, so Nymark, müssen Geschichten dazu immer wieder erzählt werden, sonst werden sie vergessen und verschwinden (2000: 49).

Geschichten schaffen laut den Organisationsforschern Hansen/Kahnweiler (1993) auch ein effektives Vehikel, um die zwischenmenschlichen Aspekte in Organisationen zu fördern. Da Geschichten ein natürlicher Weg seien, um Ansichten über vergangene Ereignisse zu erzählen, und sie auf allen Ebenen zwischen allen Gruppen einer Organisation gehört werden können, genießen Geschichten eine hohe Akzeptanz als Mittel zur Kommunikation (1393).

Wilkins/Thompson (1991) merken an, dass Geschichten eine Art Landkarte des sozialen Lebens in einer Organisation seien. Auf einfache und nachvollziehbare Weise könne über Geschichten vermittelt werden, wie die zwischenmenschlichen Dinge in Organisationen gehandhabt werden.

In Geschichten manifestiert sich daneben auch die Einzigartigkeit eines Unternehmens, in Abgrenzung zu anderen Organisationen. Martin et al. (1983) stellten bei ihren Untersuchungen fest, dass die in Organisationen kursierenden Geschichten in den Augen der Betrachter die Einzigartigkeit ihrer Organisation widerspiegeln und die Annahme bestärken würde, dass keine Organisation wie die eigene sei. Durch das Erzählen von Geschichten werde in Unternehmen versucht, diese Einzigartigkeit herauszustellen, auch wenn diese de facto gar nicht vorhanden sei bzw. wenn der Inhalt der Geschichte keine eindeutige Abgrenzung zu anderen Organisationen darstelle (vgl. auch Martin 1992, Wilkins 1983).

Als eine weitere Funktion von Geschichten in Organisationen kann die Definition und Festigung des sozialen Standes, der Seniorität und der Kompetenz von Mitarbeitern innerhalb einer Gruppe angesehen werden (Orr 1996: 126). Daneben kann mittels erzählter Geschichten, z. B. über erfolgreiche Problemlösungen, auch eine Erhöhung des Status erfolgen (Van Maanen 1988). Denn ohne Geschichten, die in der Organisation verbreitet werden, erfahren Kollegen nichts von erzielten Erfolgen und bringen diese nicht mit entsprechenden Personen in Zusammenhang.

Einen Schritt weiter gingen Weick/Browning (1986), indem sie behaupteten, dass durch Geschichten Werte, Kultur und Einstellungen in Organisationen nicht nur vermittelt bzw. gefestigt werden könnten (siehe z. B. Schein 1985), sondern dass diese erst durch Geschichten kreiert würden: „Stories are not a symptom of culture, culture is an symptom of storytelling" (251).

Auch Boyce (1995) geht davon aus, dass Geschichten eine effektive Methode sind, wenn es darum geht, kollektive Bedeutungen unter Mitarbeitern zu konstruieren.

Vor allem auch der Organisationspsychologe Weick (1995) spricht Geschichten eine große Rolle bei der Konstruktion von Sinn und Bedeutung in Organisationen zu. Weick geht dabei davon aus, dass der Mensch in Erzählungen denke. Im Gegensatz dazu seien Organisationsmodelle aber meist auf Beweisführungen aufgebaut. Will man die Hintergründe organisationalen Lebens ergründen, müsse man Erzählungen als Interpretationshilfe heranziehen. Geschichten lieferten 
dabei wichtige Inhalte für die Sinnstiftung in Organisationen, denn mit ihnen werden die in einem kleineren Zusammenhang gewonnenen Bedeutungen artikuliert und transportiert. Über ein Repertoire an miteinander verflochtenen Geschichten bildeten sich schließlich Sinn und Bedeutung einer Organisation heraus (127). Im Gegensatz zu den meisten Autoren erwähnt der Organisationstheoretiker Gabriel (2000) aber auch die Möglichkeit von Geschichten, Sinn und Bedeutungen in Organisationen zu untergraben bzw. zu zerstören. Dies sei z. B. der Fall, wenn Geschichten sich widersprechen würden (5).

Nymark fügt hinzu, dass Geschichten über zukünftige Veränderungsprozesse in Organisationen eine deutlich geringere Bedeutung als Geschichten über bereits stattgefundene, dramatische Ereignisse zukomme. Er sieht Geschichten in Unternehmen als sozial konstruierte und ausgehandelte Abschlüsse vergangener Ereignisse an. Das Erzählen von Geschichten könne dabei als eine Art kognitives sense-making-tool betrachtet werden, mit dem Interessensgruppen in Organisationen gemeinsam bedeutende Ereignisse der Vergangenheit in einem fortlaufenden dynamischen Prozess reinterpretierten (2000: 47).

Neben der Bindung an eine Organisation und der Stärkung des sozialen Zusammenhalts tragen Geschichten auch maßgeblich zur Bewahrung und zum Transport der Kultur einer Organisation bei.

Für den Ethnologen Götz (2001) lässt sich mittels Geschichten und kursierender Anekdoten die inoffizielle Kultur einer Organisation aufzeigen, d. h. all jene Aspekte des Arbeitsalltags, die in einer Beziehung zur offiziellen Kultur der Organisation stehen, sich von dieser aber auch ganz unabhängig entwickelt haben können (219). Erzählungen können hier aussagekräftige Indikatoren für individuelle und kollektive Sichtweisen der informellen Betriebskultur sein. Auch Weick (1995) spricht davon, dass sich mit Geschichten kulturelle Merkmale ans Licht holen ließen, die sonst eher im Dunkeln bleiben bzw. die schwierig zu artikulieren seien (129).

Aber, so merkt Wilkins (1983) an, mittels Geschichten könnten nicht nur unausgesprochene kulturelle Merkmale geteilt werden, sondern mit ihnen können auch Richtlinien und Hilfestellungen für das Treffen von wichtigen Entscheidungen vermittelt werden. Wilkins führt hierfür das Beispiel eines Managers an, der, während er im Fernen Osten an einem Projekt arbeitete, eine wichtige Entscheidung fällen musste und seinen Vorgesetzten im ,Headquarter' nicht erreichen konnte. Er setzte sich dann mit seinem Team zusammen und überlegte, was der Präsident des Unternehmens in so einem Fall wohl getan hätte. In diesem Rahmen stellte der Manager eine Geschichte vor, die ihm der Präsident einmal erzählt hatte. Diese wurde zur Grundlage für die zu treffende Entscheidung. Das Topmanagement des Unternehmens stimmte dieser Entscheidung später in allen Punkten voll zu (85).

Darüber hinaus sind Geschichten auch eine wichtige Orientierungshilfe für neue Mitarbeiter, um mehr über die Kultur und innere Logik einer Organisation zu erfahren. 
So wenden sich neue Mitarbeiter mit ihren Fragen oftmals zunächst an andere Mitarbeiter, bevor sie zu Vorgesetzten gehen. Die Antworten, so fand Nymark heraus, die man den neuen Mitarbeitern dann gibt, werden oftmals in kleine Geschichten über vergangene Ereignisse innerhalb der Organisation verpackt. Die Geschichten über die Vergangenheit bringen für neue Mitarbeiter die Gegenwart dann in einen verständlichen Kontext und geben ihnen Bedeutung (2000: 56). Dies führt zu einer Orientierungsgrundlage für die Zukunft.

Geschichten können aber auch dazu dienen, Change-Prozesse in Organisationen zu steuern. So betont Nymark, dass durch das Erzählen von Geschichten eine Voraussetzung für Veränderungen in Organisationen geschaffen werden kann. Auch für Browning können Geschichten eine Struktur, die organisationale Veränderungen generieren kann, beinhalten.

Auch für Boje (1991) sind organisationale Geschichten und Change-Prozesse eng miteinander verflochten. Begründet sieht er dies in der Tatsache, dass Geschichten zu einem kollektiven Dialog unter den Mitgliedern einer Organisation führten und so in einer ,gesunden" Organisation Hinweise für notwendige oder bereits stattgefundene Change-Prozesse lieferten. Dadurch haben Geschichten eine Art „Anzeigerfunktion“ für Change-Prozesse.

Stephen Denning, ein ehemaliger Manager der Weltbank, leitet anhand seiner Praxiserfahrungen aus dem Einsatz von Geschichten ab, dass im Erzählen von Geschichten sogar ein strategisch einsetzbares Tool gesehen werden kann, das dazu genutzt werden könne, organisationale Change-Prozesse zu unterstützen. Denn Geschichten ermöglichten es Mitarbeitern, sich und ihre Organisation in einem anderen Licht zu sehen. Durch die neuen Perspektiven, die Geschichten aufwerfen, könne man auch die Einstellung von Mitarbeitern zu Entscheidungen in der Organisation beeinflussen bzw. verändern (2001: 191).

Geschichten eignen sich daneben auch, Mitarbeiter von einer bestimmten Sache bzw. von einer gezielten Unternehmensphilosophie zu überzeugen und den zu verbreitenden Inhalten mehr Glaubhaftigkeit zu verleihen.

In verschiedenen Laborexperimenten untersuchten Martin/Powers (1983), inwieweit man durch den Einsatz von Geschichten die Glaubwürdigkeit einer Firmenpolitik bzw. -philosophie unterstützen kann. Ihre Ergebnisse zeigten, dass man mittels Geschichten Überzeugungen deutlich nachhaltiger transportieren kann als mit anderen Mitteln, wie z. B. Statistiken oder Berichten. Laut Martin und Powers erzeugen die Geschichten einen true believer, also eine wirkliche Überzeugung bei den Menschen. Anhand weiterer Experimente konnten sie belegen, dass Geschichten auch einen Einfluss auf die Glaubhaftigkeit einer Firmenphilosophie haben. Allerdings nur, wenn diese im Einklang zu anderen Informationen stünden. Stehen Geschichten im Widerspruch zu anderen Informationen wie Statistiken, haben sie indessen keinen großen Einfluss auf die Glaubhaftigkeit. In diesem Zusammenhang warnen sie auch vor der Möglichkeit einer corporate propaganda, vor dem bewussten Missbrauch von Geschichten, um Mei- 
nungen über die Unternehmenspolitik zu manipulieren und Personen zu überzeugen. Es dürfe nicht vergessen werden, dass Geschichten ein mächtiges Managementtool darstellten, das nicht bewusst missbraucht werden dürfe. Allerdings stellt Nymark auch fest, dass Geschichten, die für Mitarbeiter Sinn ergeben und von großer Bedeutung sind, meistens die sind, die nicht vom Management gezielt beeinflusst wurden (2000: 51).

Neben den eigenen Mitarbeitern lassen sich aber auch Kunden durch oder mit Hilfe von Geschichten, über z. B. bestimmte Produkte oder Dienstleistungen, überzeugen. Geschichten kommt daher auch eine große Bedeutung bei der Vermarktung von Produkten und Dienstleistungen zu. So ist sich der Manager von Apple Computer Ing. Erickson sicher, dass Geschichten eine große Rolle bei der Produktentscheidung von Kunden spielen. Denn mit Geschichten könne demonstriert werden, wie ein Produkt funktioniere und welchen Nutzen durch es erlangt werde und zwar in einer verständlichen und nachvollziehbaren Weise (Erickson 1995). Darum spricht er sich auch dafür aus, aktiv bei Kunden nach Geschichten zu suchen, die sie mit den Produkten erlebt haben und diese gezielt in die Vermarktung und Produktisierung einzubauen. Auch Leiber (1997), sieht in ,wahren' Geschichten von Kunden, über Erfahrungen mit Produkten, ein gutes Marketingtool.

Eine wichtige Rolle spielen Geschichten auch bei der Vermittlung und Speicherung von Wissen in Organisationen. Da sich Mitarbeiter auch im Alltagsleben Geschichten erzählen, wird dies als eine natürliche Form der Weitergabe von Wissen akzeptiert und knüpft damit an die bereits von Kindesbeinen an trainierte Form der Wissensaufnahme an (vgl. Mishler 1986).

Für den Pädagogen Bruner (1990) beinhalten Erzählungen die Hauptform menschlichen Wissens. Laut seinen Ausführungen wird es erst über Erzählungen möglich, menschlichen Erfahrungen ein Muster zu geben. Der Kommunikationsforscher Fisher (1987) geht noch weiter und sieht in Geschichten sogar die Hauptform der menschlichen Kommunikation überhaupt. Begründet sieht Fisher dies in der menschlichen Gewohnheit, neues Wissen über die Interpretation, Evaluation und den Vergleich alter und neuer Geschichten zu generieren.

Auch Lerntheoretiker wie Schank (1990) stellten fest, dass Geschichten eine enorme Auswirkung auf unser Wissen und unser Gedächtnis haben. Geschichten zu hören und sie zu erzählen, forme die Erinnerung an unsere Erfahrungen. Schank sieht darüber hinaus eine enge Verbindung zwischen Intelligenz und dem Einordnen von Geschichten. Er behauptet gar: „Knowledge is [s]tories“ (1) und dass das menschliche Gedächtnis anhand von Geschichten aufgebaut sei. Nicht jede Erfahrung gäbe eine gute Geschichte ab, aber wenn sie es täte, fiele es uns viel leichter, uns an sie zu erinnern. Die Gedächtnisforschung spricht hier auch vom „episodischen Gedächtnis“ (vgl. auch Fuchs 2009, Spitzer 2007).

Zur Vermittlung von Wissen in Fachbüchern werden Geschichten schon seit längerem als Ergänzung zur theoretischen Beschreibung herangezogen. Und sys- 
tematisch werden auch immer häufiger Geschichten bei Trainings und Weiterbildungen in Organisationen eingesetzt. Die Informatikerin Neal (2001) spricht sich darüber hinaus dafür aus, im Rahmen von E-Learning mehr mit Geschichten zu arbeiten.

Bei dieser Entwicklung werden Geschichten nicht nur als informelle, nicht strukturierte Kommunikationsmöglichkeit angesehen, sondern das Erzählen von Geschichten sollte, so der Managementforscher Vance, gezielt für effektive Trainingsmaßnahmen eingesetzt werden (1991: 52).

Die Ökonomen Pennington/Hastie (1992) untersuchten im Rahmen einer Studie die Rolle von Geschichten bei der Findung richterlicher Entscheidungen. Sie stellten fest, dass Teilnehmer der Studie jeweils jene Seite bevorzugten, deren Aussagen in Form von Geschichten vorgetragen wurden. Ähnliche Ergebnisse erzielten Adaval/Wyer (1998) bei der Untersuchung von Konsumentenverhalten.

Aber nicht nur bei der Informationsverarbeitung spielen Geschichten eine wichtige Rolle, sondern auch für das menschliche Erinnerungsvermögen. Die Lerntheoretiker Schank/Abelson (1995) beschreiben in ihrem Artikel „Knowledge and Memory: The Real Story“, dass alles wichtige Wissen, über das Menschen im Gehirn verfügten bzw. was sie hinzugewännen, auf Geschichten basiere, die um Vergangenheitserfahrungen herum konstruiert würden. Und Vance (1991) machte im Rahmen verschiedener Trainings die Erfahrung, dass das Erzählen von Geschichten einen positiven Effekt auf das individuelle Lernverhalten in Organisationen habe. Der Einsatz von Geschichten in Trainings erhöhe die Aufmerksamkeit der Lerner und schaffe ein optimales Lernklima jenseits von Langeweile. Viele Forschungsarbeiten bestätigen seit langem, dass mittels Geschichten die Aufmerksamkeit und Lernbereitschaft erhöht werden kann (z. B. Schultz 1972).

In Business Schools und Managementtrainings in den USA wird mit Geschichten schon seit längerem gearbeitet. Besonders beliebt sind dabei fiktive Geschichten. Auf sie wird immer dann gerne zurückgegriffen, wenn es um die Vermittlung von Ethik, Moral und unternehmenskulturellen Fragestellungen geht (Alvarez/Merchan 1992). Außerdem, so Shaw (1992), könne man mit (fiktiven) Geschichten die Teilnehmer von Trainings besser erreichen und sie stärker motivieren, theoretische Inhalte zu lernen, denn sie geben den theoretischen Inhalten von Kursmaterialien mehr Relevanz.

Eine besondere Rolle kommt Geschichten bei der Verbreitung und Speicherung von technischem Detailwissen zu. In seiner vielbeachteten ethnographischen Studie „Talking about Machines“ geht Orr den kommunikativen Strategien von Technikern der Firma Xerox nach, die ihr teilweise sehr kompliziertes Wissen über die Reparatur von Kopierern mittels Geschichten, sogenannten „war stories“, aufbauen und weitergeben. Unter „war stories“ versteht Orr Anekdoten und Erfahrungsberichte, die lediglich mit so viel Kontext ausgestattet werden, wie die Situation, in der sie erzählt werden, es verlangt (1996: 125). Sie bestehen dabei im Wesentlichen aus dem Namen des Technikers, der die Arbeit verrichte- 
te, der Bezeichnung der Maschine, um die es ging, das Problem, das gelöst werden sollte und dem Lösungsweg. In der Regel werden sie von der Person erzählt, die sie erlebt hat. Die „war stories“ sind dabei oft extrem unvollständig und für Außenstehende kaum als Geschichten zu erkennen. Nur im Team bekommen sie Bedeutung und grenzen somit die Welt der Techniker ab. Orr stellte fest, dass die „war stories“ den Technikern vor allem zur gemeinsamen Fehlerdiagnose bei technischen Problemen dienen. Das Zirkulieren von Geschichten unter den Kollegen sei für die Techniker aber auch eine wichtige Form der Informationsweitergabe und eines Abgleichs und Aufbaus von Wissen. Mit den Geschichten werde daneben auch die Expertise der Techniker untereinander zelebriert (5).

Neben dem technischen Detailwissen lässt sich mittels Geschichten auch das schwer fassbare ,verborgene ${ }^{6}$ oder ,implizite ${ }^{6}$ Wissen in Organisationen vermitteln. Die Organisationstheoretiker Nonaka/Takeuchi (1995) weisen in ihrem vielzitierten Buch The Knowledge-Creating Company in Anlehnung an Polanyi (1966) darauf hin, dass es zwei Typen von Wissen gibt: Zum einen das explizite Wissen („explicit knowledge“) wie es in Handbüchern und Checklisten enthalten ist. Dieses Wissen ist leicht systematisier- und speicherbar, da es außerhalb der Köpfe einzelner Personen vorliegt. Schwieriger verhält es sich mit dem zweiten Wissenstyp, dem impliziten Wissen („tacit knowledge“ - 59 ff.). Es wird durch Erfahrung erlernt und ist nur indirekt durch Metaphern und Analogien vermittelbar. Dieses Wissen ist an bestimmte Personen gebunden, die sich ihres Wissens oft selbst nicht bewusst sind. Als Grundproblem des Wissensmanagements in Organisationen nennen Nonaka und Takeuchi die Überführung von impliziten in explizites Wissen. Diesen Prozess nennen sie „Externalisierung“ (64). Denn erst wenn Wissen explizit vorliegt, ist es über einzelne Personen bzw. Personengruppen hinaus nutzbar (North 1998). Einen Weg, implizites Wissen zu externalisieren, sehen Nonaka und Takeuchi über Metaphern und Analogien, denn diese werden in der Regel in Form von Geschichten konserviert und weitergegeben. Daher tragen Geschichten zur Externalisierung und damit zum Managen von impliziten Wissen bei.

Schafft man es, das in organisationalen Geschichten enthaltene Wissen zu erfassen und aufzubereiten, so kann dieses Wissen auch eine wertvolle Ergänzung zu den gängigen Wissensmanagement-Methoden in Organisationen darstellen. Die Medienpädagogen Reinmann-Rothmeier/Vohle (2001) sehen mittels Geschichten eine Möglichkeit, an Wissen heranzukommen, das über herkömmliche Wissensmanagementmethoden wie Datenbanken, Intranetlösungen, Web 2.0Tools nicht generiert und aufbereitet werden kann.

Sie merken an, dass in Geschichten vielfältige Möglichkeiten stecken, um das Managen von Wissen in Organisationen zu bereichern (Reinmann-Rothmeier/ Vohle 2001, s. auch Reinmann-Rothmeier/Mandl 2002). So können Geschichten zum Beispiel zu mehr Wissenstransparenz beitragen, indem sie schwer vermittelbare Botschaften und Werte in einer ,narrativen Verpackung' darstellen und er- 
möglichen, multiple Perspektiven darzustellen. Damit dienen Geschichten der Repräsentation von Wissen.

Geschichten können auch die Handlungsfähigkeit erböhen, indem sie durch die Darstellung von nachvollziehbaren Handlungsschritten z. B. dazu beitragen, gesammelte Erfahrungen in anderen Kontexten zu nutzen. Damit tragen Geschichten zur Nutzung von Wissen bei.

Eine weitere Funktion ist die Erböbung der Kommunikationskultur durch Geschichten. Da über Geschichten auch tabuisierte Themen, heimliche Spielregeln oder verschwiegene Problemursachen angesprochen und ausgetauscht werden können, fördern sie ein lernfreundliches und offenes Klima und tragen zur Kommunikation von Wissen bei.

Darüber hinaus haben Geschichten das Potential, die Innovationsbereitschaft bei Erzählern und Zuhörern zu erhöhen. Denn mittels Geschichten werden kreatives und produktives Denken gefördert und Zukunftsentwürfe generiert. Somit können Geschichten sogar zur Generierung von Wissen beitragen.

Die hier aufgezeigten unterschiedlichen Rollen und Funktionen von Geschichten zeiegn, dass organisationale Geschichten bereits eine Vielzahl von Funktionen in Organisationen einnehmen bzw. einnehmen könnten, wenn man sie bewusst einsetzen würde und dass in Geschichten ein vielschichtiges Potential für Organisationen schlummert (Thier 2003). Im Folgenden wird eine Methode vorgestellt, die genau hier ansetzt und gezielt mit den Erfahrungen und Geschichten von Mitarbeitern über bestimmte Themenbereiche arbeitet.

\section{Die Storytelling-Methode des M.I.T.}

Wer „Storytelling in Unternehmen“ in die Suchmaschinen des Internets eingibt, wird schnell feststellen, dass sich unter diesem Begriff in den letzten Jahren eine Vielzahl von ganz unterschiedlichen Ansätzen, Methoden, aber auch Sichtweisen finden lassen. Die Spannbreite reicht dabei vom Einsatz von Geschichten in Konferenzen, um Mitarbeiter für eine neue Idee zu begeistern, über die Nutzung von Geschichten für überzeugendere Präsentationen, dem Kreieren von Geschichten im Rahmen von Trainings bis hin zur Erhebung schwer zugänglichen Wissens mittels Geschichten. In den seltensten Fällen sind diese Ansätze und Methoden näher wissenschaftlich betrachtet worden. Eine Ausnahme bildet die am Massachusetts Institute of Technology (MIT), USA, Mitte der 1990er Jahre entwickelte Storytelling-Methode, die bereits in unterschiedlichsten Kontexten und Unternehmen erfolgreich eingesetzt wurde und darüber hinaus in gut dokumentierter Form vorliegt. Sie soll hier näher vorgestellt werden.

Wie können kollektive Erfahrungen von Mitarbeitern aus der Vergangenheit in Organisationen so genutzt werden, dass es in der Zukunft nicht zu Wiederholungsfehlern kommt und Prozesse effektiver durchgeführt werden können? So lautet die Ausgangsfrage, mit der sich eine Gruppe von Forschern, Managern und 
Journalisten am Center for Organizational Learning des MIT in Cambridge im Rahmen eines Forschungsprojektes zur Enträtselung kollektiven Lernverhaltens beschäftigte. Ihr Ziel war es, ein tool zu entwickeln und zu testen, das es ermöglicht, Erfahrungen über zentrale Ereignisse in Organisationen aus unterschiedlichen Perspektiven zu erfassen und aufzubereiten.

Das von ihnen entwickelte Tool nannten sie Learning History, zu Deutsch ,Erfahrungsgeschichte'. Im Folgenden wird dieser methodische Ansatz StorytellingMethode genannt werden (Kleiner/Roth 1998).

Bereits 1997 gab es am MIT mehr als 15 Projekte, die die Storytelling-Methode einsetzten und mit ihr experimentierten. Vor allem in großen amerikanischen Unternehmen wurde die Methode in verschiedenen Einsatzkontexten getestet.

Bei der Entwicklung der Storytelling-Methode griffen die Forscher vor allem auf Theorien und Techniken der Ethnographie, der Aktionsforschung und des Journalismus zurück.

Von der Ethnographie übernahmen Kleiner und Roth beispielsweise die Art und Weise der Untersuchung von (Organisations-)Kulturen. Übernommen wurden vor allem narrative Interviewtechniken und die Technik der teilnehmenden Beobachtung, um das tägliche Leben von Mitarbeitern in spezifischen Unternehmenskulturen zu untersuchen. Sie griffen dabei auf Autoren zurück wie z. B. Van Maanen (1979).

Die Aktionsforschung (vgl. z. B. Argyris 1990, 1993; Schein 1985) bot ihnen Methoden, um mit Situationen umzugehen, in denen der Forscher gleichzeitig auch in den Veränderungsprozess des Untersuchungsgegenstandes involviert ist. Vom Journalismus leiteten sie Techniken ab, wie schnell zum Kern einer Sache vorgedrungen, wichtige Fakten ausgewählt und eine Sache so aufbereitet werden kann, dass sie den Leser bzw. Zuhörer berührt. Daneben holte man sich aber auch aus der mündlichen Geschichtsforschung (oral history) und dem Theater Anregungen.

Was aber ist nun genau unter der Storytelling-Methode bzw. unter Erfahrungsgeschichten zu verstehen? Storytelling beschreibt ein methodisches Vorgehen, mit dem (Erfahrungs-)Wissen von Mitarbeitern über einschneidende Ereignisse der Vergangenheit (wie z. B. ein Pilotprojekt, eine Fusion, Reorganisationen, die Erschließung eines neuen Geschäftsfeldes oder eine Produkteinführung) aus unterschiedlichsten Perspektiven der Beteiligten erfasst, ausgewertet und in Form einer Geschichte so aufbereitet wird, dass die ganze Organisation davon profitieren kann. In Workshops werden die Inhalte anschließend im Unternehmen verbreitet und damit ein Lernprozess im Unternehmen in Gang gesetzt, der helfen soll, Wiederholungsfehler zu vermeiden und neue Lösungsansätze zu generieren. Bei einer ,Erfahrungsgeschichte' handelt es sich um das Produkt, das durch den Einsatz der Storytelling-Methode in Organisationen entsteht. Das ist eine schriftlich festgehaltene Nacherzählung wichtiger Vorkommnisse aus der jüngsten Vergangenheit einer Organisation. Der Umfang dieses Dokumentes bewegt sich zwischen ca. 25 bis 100 Seiten und ist zweispaltig aufgebaut (siehe Abbildung I). 


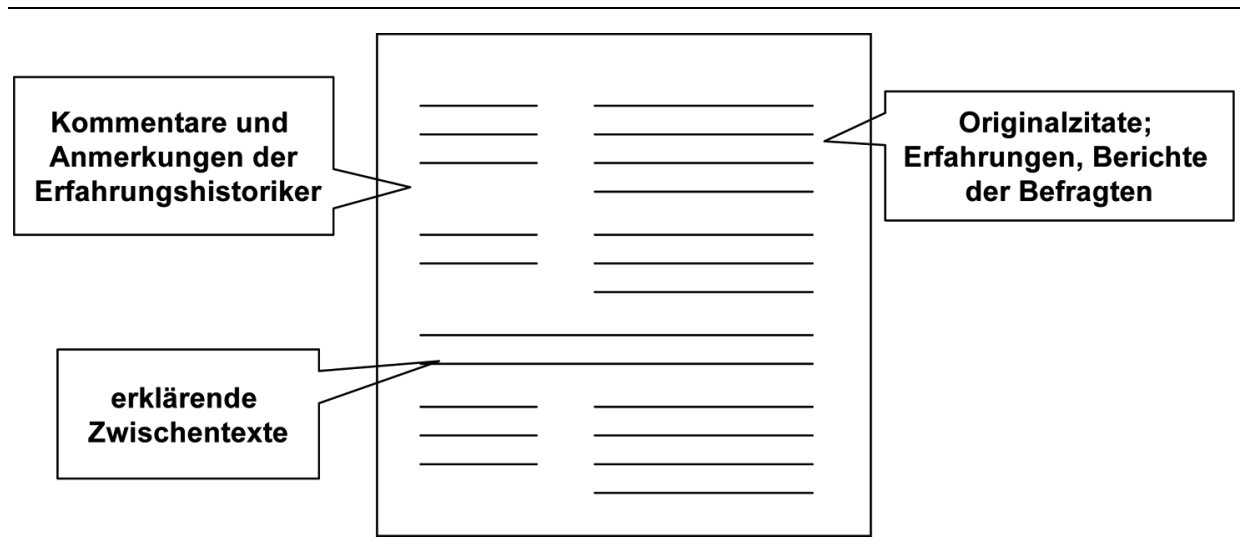

Abbildung I: Zweispaltiger Aufbau von Erfahrungsgeschichten

In der rechten Spalte wird die Geschichte mit Originalzitaten von denen erzählt, die die dargestellten Ereignisse erlebt haben. Sie berichten ihre Sicht der Ereignisse. Die Erzähler werden dabei nicht namentlich genannt, sondern bleiben anonym. Die einzelnen Zitate der unterschiedlichen Personen sind dabei so miteinander verwoben bzw. mit Zwischentexten versehen, dass eine nachvollziehbare, zusammenhängende, aber auch emotionale Geschichte entsteht.

Die linke Spalte ist dagegen mit Analysen und teilweise provokanten Kommentaren von den Schreibern der Erfahrungsgeschichte, den sogenannten „Erfahrungshistorikern" versehen. Ziel dieser Anmerkungen ist es, den Leser zum Nachdenken anzuregen und Punkte anzusprechen, die möglicherweise in den Zitaten nur angedeutet werden, aber an die Oberfläche gebracht werden sollten.

Die Erfahrungsgeschichte kann dabei in verschiedene, aufeinander aufbauende oder eigenständige Kurzgeschichten unterteilt sein. Bei der Aufbereitung sind vielerlei Formen denkbar und auch multimediale Elemente können eingesetzt werden. Um mit der Erfahrungsgeschichte möglichst effizient arbeiten zu können, bekommen die Leser in einer Art Anleitung, bevor die eigentliche Erfahrungsgeschichte beginnt, Hinweise zum Lesen der Geschichte. Daneben werden in der Regel auch der Kontext des untersuchten Projektes, die befragten Personen und die Ziele, die die Organisation mit der Erfahrungsgeschichte erreichen will, für den Leser erläutert. Die Erfahrungsgeschichte und diese erläuternden Zusätze ergeben dann das fertige Erfahrungsdokument, das in der Organisation verbreitet werden kann.

Das Ziel ist in erster Linie nicht das Schreiben der Erfahrungsgeschichte bzw. die Erfahrungsgeschichte an sich, die von Mitarbeitern gelesen und in den Schrank gestellt werden kann, sondern die während der Erstellung und Verbreitung der Geschichten ablaufenden Prozesse in der Organisation, wie z. B. die Reflexion von Ereignissen, Gruppendiskussionen, das Erzielen von Erkenntnissen und das Ableiten von Verbesserungsideen, d. h. das Gewinnen eines tieferen Verständnisses für bestimmte Ereignisse und die Übertragung der dort gemachten Erfahrungen auf neue, zukünftige Handlungen. 
Mit dem Einsatz der Storytelling-Methode kann man in Unternehmen zahlreiche Effekte erzielen: Der wichtigste Effekt ist nach Kleiner und Roth das Bilden von Vertrauen unter den Mitarbeitern mittels Erfahrungsgeschichten. So bekommen Mitarbeiter, die bislang das Gefühl hatten, isoliert zu sein oder deren Meinung bisher ignoriert wurde, die Chance, sich zu äußern. Die Workshops und Diskussionen über das Erfahrungsdokument führen zu einer kollektiven Reflektion über entscheidende Ereignisse, was den Mitarbeitern ermöglicht, über ihre eigenen Annahmen und Ängste zu sprechen und Vertrauen zueinander aufzubauen. Und wenn das Vertrauen untereinander wächst, so Kleiner und Roth, dann kann sich in Organisationen auch eine Umgebung entwickeln, die ein gemeinsames Lernen ermöglicht.

Ein weiterer Effekt ist, dass Themen zur Sprache kommen, über die sonst nicht offen gesprochen wird, die aber wichtig sind und einen großen Einfluss auf die Prozesse innerhalb von Organisationen haben. Die Erfahrungsgeschichte mit ihren anonymen Zitaten und provokanten Anmerkungen von Außenstehenden ermöglicht es, diese Themen aufzudecken, ohne einzelne Personen bloßzustellen.

Daneben kann mit Storytelling erfolgreich Wissen von einer Gruppe von Mitarbeitern zu einer anderen transferiert werden. Anstatt einfach "Erfolgsbeispiele und ,Lessons Learned' zu kopieren, die nichts mit dem realen Arbeitsalltag zu tun haben, können die Leser von Erfahrungsgeschichten etwas über Hintergründe und Impulse erfahren, die zu bestimmten Situationen und Reaktionen darauf in ihrer Organisation führten. Die gewonnenen Einsichten lassen sich so viel einfacher auf die eigene Situation übertragen.

Darüber hinaus helfen Erfahrungsgeschichten, einen Grundstock an übertragbarem Wissen in Organisationen zu generieren. Auch wenn sich die Inhalte der Geschichten über ein bestimmtes Ereignis bzw. Projekt ranken, so lassen sich die Lehren doch laut Kleiner und Roth, auf zahlreiche Projekte und Prozesse übertragen. Für Lehner (2000) ist die Storytelling-Methode daher auch als ein instrumenteller Ansatz zur Umsetzung organisatorischen Lernens zu sehen (202).

Mit Storytelling können aber auch Veränderungsprozesse in Organisationen angeregt werden, denn über die Erfahrungsgeschichte wird ein Dialog über Prozesse und Verhaltensweisen innerhalb der Organisation unter den Mitarbeitern angestoßen, der Stoff für Veränderungen bietet.

Kleiner und Roth sind der Meinung, dass durch den Einsatz der StorytellingMethode so viele Effekte in Organisationen erzielt und angeregt werden könnten, dass diese Methode in Zukunft standardmäßig bei der Ausbildung von Managern gelehrt werden müsste. In der Praxis gliedert sich der Ablauf eines Storytelling-Prozesses in fünf aufeinander aufbauende Phasen, die hier kurz erläutert werden sollen (siehe neben Kleiner/Roth 1998 auch Thier 2003, 2010):

1. Planungsphase: In der Planungsphase geht es vor allem darum, den groben Rahmen des Methodeneinsatzes in der Organisation abzustecken. Dazu wird zunächst ein Team von „Erfahrungshistorikern“ gebildet. Dieses besteht in 
der Regel aus externen Beratern und Mitgliedern der Organisation, die bereit sind, die externen Berater während der Durchführung der StorytellingMethode zu unterstützen. Dieses Team sucht in der Organisation nach sogenannten „noticeable results“, d. h. nach zentralen Ereignissen, Projekten und Vorkommnissen der jüngsten Vergangenheit, die es wert sind, näher untersucht zu werden. Daneben legt man die Zielgruppen fest, die später von der erstellten Erfahrungsgeschichte profitieren sollen. Auch müssen in dieser Phase alle Vertrags- und Budgetfragen geklärt werden. Kleiner und Roth sehen in der Planungsphase den Schlüssel für das Gelingen des Storytelling-Prozesses, da hier die Richtung der späteren Erfahrungsgeschichte bestimmt wird.

2. Intervierephase: Den Kern der nächsten Phase bilden die in der Organisation zu führenden Interviews. Dabei werden je nach Projekt zwischen ca. 10 und 50 Personen interviewt. Wichtig ist, dass so viele unterschiedliche Perspektiven wie möglich auf die zu untersuchenden Ereignisse eingefangen werden, das heißt, vom Praktikanten über den Projektleiter bis zum Geschäftsführer werden möglichst viele Beteiligte interviewt. Auch die Außenperspektive wird berücksichtigt, d. h. auch Kunden, Lieferanten und Berater werden befragt. Während des Interviews wird den Interviewten die Möglichkeit gegeben, möglichst frei über ihre Erlebnisse und Eindrücke zu erzählen. Das Interview findet daher ohne festgeschriebenen Leitfaden statt. Daneben wird in dieser Phase aber auch in Dokumenten und Projektunterlagen nach weiteren Hinweisen über das zu untersuchende Ereignis gesucht. Die Planungs- und Interviewphase gehen in der Praxis recht nahtlos ineinander über und bilden keine scharfe Trennlinie.

3. Extrabierphase: Jetzt geht es daran, „the mass of data“, wie Kleiner und Roth sagen, also die Masse an Interviewdaten, auszuwerten und zu systematisieren. Dazu werden alle Interviews zunächst vollständig transkribiert. Anschließend suchen die Erfahrungshistoriker innerhalb der vorhandenen Materialien (Interviews und Dokumente) nach bedeutenden Themen, aussagekräftigen Zitaten und sich widersprechenden Aussagen und ordnen diese erkennbaren Themengruppen zu. Der Prozess der Auswertung ist dabei an das von Strauss/ Corbin (1990) entwickelte Konzept der „grounded theory“ (23) angelehnt, das besagt, dass alle Interpretationsversuche immer wieder mit den vorhandenen Datenmaterialien belegt werden müssen, bis sich unter den Forschenden eine gemeinsame Theorie erkennen lässt. Außerdem unterliegt die Auswertungsphase streng den drei Imperativen der Storytelling-Methode (siehe unten). Am Ende dieser Phase liegt eine Art ,Rohmaterial' für die Erfahrungsgeschichte vor.

4. Schreibphase: In dieser Phase wird die eigentliche Erfahrungsgeschichte geschrieben. Bei der Gestaltung lehnen sich Kleiner und Roth an das anthropologische Konzept der ,jointly told tale“ an, d. h. von Forschern und Erforschten gemeinsam erzählten Geschichte (Van Maanen 1988). Beteiligte und Er- 
fahrungshistoriker erzählen hier gemeinsam von den untersuchten Ereignissen, das heißt, aus den Originalzitaten und den Anmerkungen der Erfahrungshistoriker wird eine wahre, aussagekräftige, nachvollziehbare und spannende Geschichte gewoben. Um die Aussagen optisch voneinander abzugrenzen, wird die oben beschriebene Spaltenform gewählt. Kleiner und Roth merken an, dass für diesen Schritt die Erfahrungshistoriker viel Training und Erfahrung benötigen.

5. Validierungsphase: Um sicherzugehen, dass die Inhalte der anschließend in der Organisation zu verbreitenden Erfahrungsgeschichte auch wirklich sinngemäß wiedergegeben wurden und alle Beteiligten mit der Darlegung ihrer Aussagen einverstanden sind, muss die Geschichte jetzt validiert werden. Bevor Irgendjemand in der Organisation die Erfahrungsgeschichte zu sehen bekommt, werden allen Interviewpartnern ihre Zitate zur Gegensicht zugesandt, wobei die Möglichkeit besteht, Aussagen zu streichen oder zu korrigieren. Damit sollen die Anonymität und die Akzeptanz der Interviewpartner gesichert werden. Anschließend wird in einem „validation workshop" die fertige Erfahrungsgeschichte einer ausgewählten Gruppe an Beteiligten und anderen Mitarbeitern vorgelegt. Dies ermöglicht einen Test der Erfahrungsgeschichten in einem größeren Kontext. Nach dem Workshop werden ggf. weitere Änderungen vorgenommen.

6. Verbreitungsphase: In gut vorbereiteten Workshops wird die fertige Erfahrungsgeschichte nun in der Organisation verbreitet. Mitarbeiter aus allen Bereichen sollen dabei mit dem Erfahrungsdokument vertraut gemacht werden und aus den dort formulierten Erfahrungen und Lehren lernen. So soll im ganzen Unternehmen nach und nach ein Lernprozess in Gang gesetzt und die Erfahrungen nutzbringend verbreitet werden (Kleiner/Roth 1998).

\section{Extrahieren}

\section{Schreiben}

\section{Interviewen}

6. Verbreiten

5. Validieren

\section{Planen}

Abbildung II: Phasen des Storytelling-Prozesses

Lernende Organisation 
Während des Durchlaufs des gesamten Storytelling-Prozesses, aber besonders bei der Auswertung der Daten und der Erstellung der Erfahrungsgeschichten müssen nach Kleiner und Roth immer wieder folgende drei Imperative berücksichtigt werden:

1. „The research imperative" - Damit ist das saubere wissenschaftliche Arbeiten und das sichtbare Trennen von Fakten, Zitaten, Hypothesen und Interpretationen gemeint.

2. „The pragmatic imperative“ - Mit diesem Imperativ soll sichergestellt werden, dass die Erfahrungsgeschichte auch so aufgebaut und geschrieben wird, dass sie von den Lesern akzeptiert wird und einen größtmöglichen Lerneffekt im Unternehmen anstößt.

3. „The mythic imperative“ - Hierunter ist die Berücksichtigung des Unternehmenskontextes zu verstehen, in den die Erfahrungsgeschichte eingebettet sein muss, und das Erzeugen einer Spannung für den Leser.

Diese drei Imperative gelten prinzipiell als Leitlinien und Hilfestellungen während der Methodendurchführung.

Der hier beschriebene Storytelling-Prozess des MIT ist sicherlich ein recht aufwändiges Verfahren, welches neben einem hohen zeitlichen Faktor auch viele Personalressourcen erfordert. Daher ist die Durchführung eher für besondere (Wissens-)Themen und Projekte in Unternehmen geeignet (Thier 2003). In vereinfachter Form wird dieser Storytelling-Ansatz jedoch mittlerweile erfolgreich in ganz unterschiedlichen Unternehmenskontexten eingesetzt. Neben den klassischen Einsatzgebieten Wissens- und Changemanagement wird besonders in den Themenfeldern Employer Branding, Recruiting, Marketing und PR immer mehr die Kraft von authentischen Unternehmensgeschichten erkannt. Auch für die interne Teamentwicklung und das Coaching von Teams in Konfliktsituationen lässt sich der Einsatz von Storytelling verstärkt beobachten (Thier 2010, Thier/Erlach 2013).

In Zukunft wird Storytelling für Unternehmen sicher noch deutlich an Bedeutung gewinnen, vor allem auch was die Bereiche Markenbildung und Öffentlichkeitsarbeit betrifft. Laut einer Aussage von Michael D. Rhodin, Senior Vice President der IBM Watson Group und ein Vordenker im Bereich Knowledge Management, heißen die Zukunftskompetenzen, in die junge Menschen heute investieren sollten: Mathematik und Storytelling! Laut ihm wird die Fähigkeit, Geschichten zu erzählen, immer wichtiger werden (Dobiéy 2014). Der Grund darin ist sicher auch als eine Reaktion auf das neue, komplizierte Kommunikations- und Informationsumfeld, in dem wir heute leben, zu verstehen. In einer Welt, in der wir mittels Google, sozialer Netzwerke und Communities immer schneller an Informationen herankommen und mit diesen überschüttet werden, wird das Ringen um Aufmerksamkeit für Unternehmen zu einem wichtigen Gut. Und wer in Blogs, auf Facebook, Twitter und Co. die beste Geschichte über sein Unternehmen, seine Kunden, Produkte und Erfahrungen erzählen kann, wird geliked', 
,retweeted' und kommentiert. Unternehmen werden sich künftig daher immer stärker damit auseinandersetzen müssen, wie sie sich von der Konkurrenz abheben und attraktive Inhalte mit magnetischer und viraler Wirkung entwickeln können. Eine Prognose dabei lautet: Erfolgreiche Marken werden sich durch Storytelling abheben.

\section{Literatur}

Adaval, R., und R. S. Wyer (1998) „The Role of Narratives in Consumer Information Processing“. Journal of Consumer Psychology 7.3: 207-45.

Alvarez, J. L., und C. Merchan (1992) „The Role of Narrative Diction in the Development of Imagination for Action“. International Studies of Management $\mho \sigma$ Organization 22.3: 27-45.

Argyris, C. (1990) Overcoming Organizational Defenses. New York: Pretice-Hall.

- (1993) Knowledge for Action. San Francisco: Jossey Bass.

Boje, D. M. (1991) „Consulting and Change in the Storytelling Organisation“. Journal of Organizational Change Management 4.3: 7-17.

- (1994) „Organizational Storytelling. The Struggles of Pre-Modern, Modern and Postmodern Organizational Learning Discourses“. Management Learning 25: 433-61.

Bonsen, M. (2000) „Eine neue Geschichte erzählen: Spirit, Mythen, Großgruppen-Interventionen und liturgische Systeme“. Das Feuer großer Gruppen: Konzepte, Designs, Praxisbeispiele für Großveranstaltungen. Hgg. M. Königswieser und M. Keil. Stuttgart: Klett-Cotta. 85-99.

Boyce, M. E. (1995) „Collective Centring and Collective Sense-Making in the Stories and Storytelling of One Organization“. Organizations Studies 16.3: 107-37.

Browning, L. D. (1991) „Organisational Narratives and Organisational Structure“. Journal of Organizational Change Management 4.3: 59-67.

Bruner, J. (1990) Acts of Meaning. Cambridge: Harvard Univ. Press.

Czarniawska, B. (1998) A Narrative Approach to Organizations Studies. Thousand Oaks: Sage.

Denning, S. (2001) The Springboard: How Storytelling Ignites Action in Knowledge-Era Organizations. Woburn: Butterworth-Heinemann.

Dobiéy, D. (2014) „As We Might Work - Die Zukunft von Wissensmanagement“. Zukunft der Wissensarbeit: Kongressband zur KnowTech 2014. Hgg. M. Arns, J. Bentele, J. Niemeier, P. Schütt und M. Weber. Berlin: GITO Verlag. 151-66.

Erickson, T. (1995) „Notes on Design Practice: Stories and Prototypes as Catalysts for Communication“. <http://www.pliant.org/personal/Tom_Erickson/ Stories.html > (Zugriff: 20. Nov. 2014).

Fisher, W. R. (1987) Human Communication as Narration: Toward a Pbilosophy of Reason, Value and Action. Columbia: Univ. of South Carolina Press. 
Fuchs, W. T. (2009) Warum das Gehirn Geschichten liebt: Mit den Erkenntnissen der Neurowissenschaften zu zielgruppenorientiertem Marketing. München: Haufe.

Gabriel, Y. (2000) Storytelling in Organizations: Facts, Fiction and Fantasies. Oxford: Univ. Press.

Götz, I. (2001) „Empirische Erhebungen in Industriebetrieben und bürokratischen Organisationen“. Methoden der Volkskunde: Positionen, Quellen, Arbeitsweise der Europäischen Ethnologie. Hgg. S. Göttsch und A. Lehmann. Berlin: Ethnologische Paperbacks. 213-33.

Hansen, C. H., und W. M. Kahnweiler (1993) „Storytelling: An Instrument for Understanding the Dynamics of Corporate Relationships“. Human Relations 46.12: 1391-1409.

Kleiner, A., und G. Roth (1998) „Wie sich Erfahrungen in der Firma besser nutzen lassen". Harvard Business Manager 5: 9-16.

Leiber, R. B. (1997) „Storytelling: A New Way to Get Close to Your Customer“. Fortune 3: 102-98.

Lehner F. (2000) Organizational Memory. München: Carl Hanser.

Martin, J. (1992) Cultures in Organizations: Three Perspectives. New York: Oxford Univ. Press.

Martin, J., und M. Powers (1983) „Organizational Stories: More Vivid and Persuasive Than Quantitative Data“. Psychological Foundations of Organizational Behaviour. Hg. B. Staw. Glenview, IL: Scott Foresman. 161-68.

Martin, J., M. Feldman, M. Hatch und S. Sitkin (1983) „The Uniqueness Paradox in Organizational Stories“. Administrative Science Quarterly 28: 438-53.

Mishler, E. G. (1986) Research Interviewing: Context and Narrative. Cambridge: Harvard Univ. Press.

Mitroff, I. I. (1983) Stakeholders of the Organizaional Mind: Toward a New View of Organizational Policy Making. San Francisco: Jossey-Bass.

Neal, L. (2001) „Storytelling at a Distance“. eLearn Magazine. <http://elearnmag. acm.org/featured.cfm?aid=566979> (Zugriff: 20. Nov. 2014)

Nonaka, I., und H. Takeuchi (1995) The Knowledge-Creating Company. Oxford: Oxford Univ. Press.

North, K. (1998) Wissensorientierte Unternehmensfübrung: Wertschöpfung durch Wissen. Wiesbaden: Gabler.

Nymark, S. R. (2000) Organizational Storytelling: Creating Enduring Values in a Hightech Company. Hinnerup: Ankerhus.

Orr, J. E. (1996) Talking about Machines: An Ethnography of a Modern Job. New York: Cornell Univ. Press.

Owen, H. (1987) Spirit: Transformation and Development in Organizations. Potomac, MD: Abbott.

Pennington, N., und R. Hastie (1992) „Explaining the Evidence: Testing the Story Model for Juror Decision Making". Journal of Personality and Social Psychology 62: 182-206. 
Peters, T., und R. H. Waterman Jr. (1982) In Search of Excellence: Lessons from America's Best Run Companies. New York: Harper \& Row.

Polanyi, M. (1966) The Tacit Dimension. New York: Anchor Day Books.

Reinmann-Rothmeier, G., und F. Vohle (2001) „Was Schiedsrichter, Manager und Rotkäppchen gemeinsam haben: Mit Geschichten Wissen managen“. Zeitschrift für Fübrung und Organisation 5: 293-300.

- (2002) „Pädagogisch-psychologische Ideen für die Repräsentation und Kommunikation von Wissen im Netz - ein narrativer Ansatz". Bild, Medien, Wissen: Visuelle Kompetenz im Medienzeitalter. Hgg. H. D. Huber, B. Lockemann und M. Scheibel. München: kopaed. 337-62.

Reinmann-Rothmeier, G., und H. Mandl (2002) „Das unausgesprochene Problem des impliziten Wissens im Wissensmanagement". Grundlagen der Weiterbildung 1: 11-14.

Schank, R. C. (1990) Tell Me a Story. A New Look at Real and Artificial Memory. New York: Collier Macmillan.

Schank, R. C., und R. P. Abelson (1995) „Knowledge and Memory: The Real Story“. Advances in Social Cognition 8. Hg. R. S. Wyer. Hillsdale: Erlbaum. 1-85.

Schein, E. H. (1985) Organizational Culture and Leadership. San Francisco: JosseyBass.

Schultz, C. (1972) „A System of Cognitive Stimulation in Instructional Strategies“. Instructional Science 1: 313-42.

Shaw, G. (1992) „Using Literature to Teach Ethics in the Business Curriculum“. Journal of Business and Technical Communications 6.2: 187-99.

Spitzer, M. (2007) Lernen: Gehirnforschung und die Schule des Lebens. Heidelberg: Spektrum.

Strauss, A. L., und J. Corbin (1990) Basics of Qualitative Research: Grounded Theory Procedures and Techniques. Newbury Park: Sage.

Thier, K. (2003) Die Entdeckung des Narrativen für Organisationen: Entwicklung einer effizienten Story Telling-Methode. Hamburg: Dr. Kovač.

- (2010) Storytelling - Eine Methode für das Change-, Marken-, Qualitäts- und Wissensmanagement. Heidelberg: Springer.

Thier, K., und C. Erlach (2013) „Der Storytelling-Prozess. Narrative Methoden zur Reflexion und Präventio von Teamkonflikten“. Konfliktdynamik 4: 272-81.

Van Maanen, J. (1979) „The Fact and Fiction in Organizational Ethnography“. Administrative Science Quarterly 24: 539-50.

- (1988) Tales of the Field: On Writing Ethnography. Chicago: Univ. of Chicago Press.

Vance, C. M. (1991) „Formalising Storytelling in Organisations: A Key Agenda for the Design of Training“. Journal of Organizational Change Management 4.3: 52-58.

Weick, K. E., und L. D. Browning (1986) „Argument and Narration in Organizational Communication“. Administrative Science Quarterly 12: 243-59.

Weick, K. E. (1995) Sensemaking in Organizations. London: Sage. 
Wilkins, A. L. (1983) „Organizational Stories as Symbols Which Control the Organization“. Organizational Symbolism. Hgg. L. R. Pondy, P. J. Frost, G. Morgan und T. C. Dandridge. Greenwich, Connecticut: Jai Press. 69-92.

Wilkins, A. L., und M. P. Thompson (1991) „On Getting the Story Crooked (and Straight)“. Journal of Organizational Change Management 4.3: 18-26. 
